BNL-114544-2017-IR

CBETA/011

April 2017

\title{
Transverse forces in the first girder magnets
}

\author{
S. Brooks
}

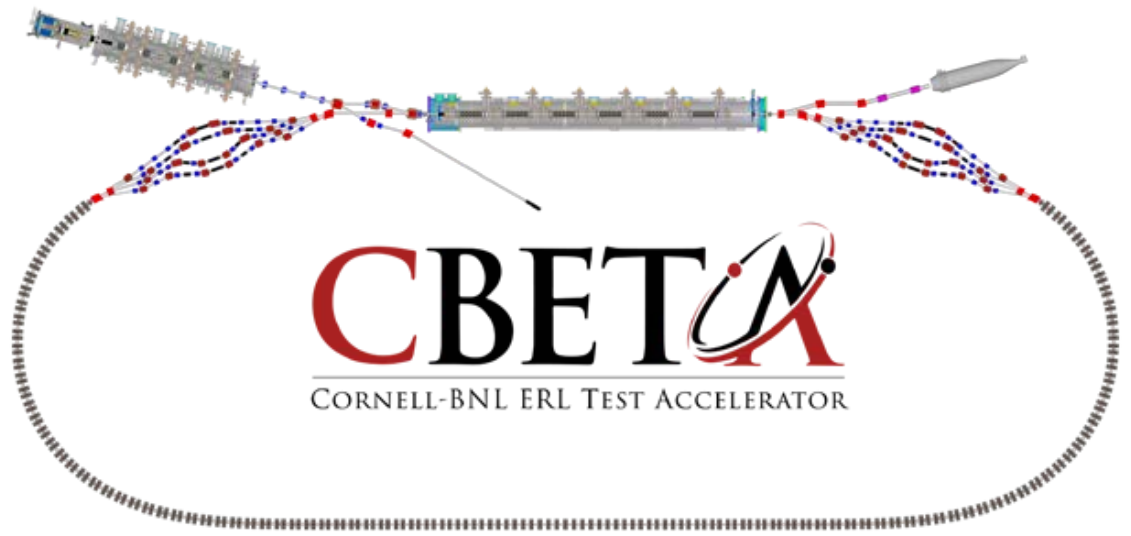

Collider-Accelerator Department, Brookhaven National Laboratory, Upton NY 11973

U.S. Department of Energy

Office of Science, Office of Nuclear Physics

Cornell Laboratory for Accelerator-Based Sciences and Education, Ithaca, NY 14850

Funded by NYSERDA contract 102192

Notice: This document has been authorized by employees of Brookhaven Science Associates, LLC under Contract No. DE-SC0012704 with the U.S. Department of Energy. The United States Government retains a non-exclusive, paid-up, irrevocable, world-wide license to publishor reproduce the published form of this document, or allow others to do so, for United States Government purposes. 


\section{DISCLAIMER}

This report was prepared as an account of work sponsored by an agency of the United States Government. Neither the United States Government nor any agency thereof, nor any of their employees, nor any of their contractors, subcontractors, or their employees, makes any warranty, express or implied, or assumes any legal liability or responsibility for the accuracy, completeness, or any third party's use or the results of such use of any information, apparatus, product, or process disclosed, or represents that its use would not infringe privately owned rights. Reference herein to any specific commercial product, process, or service by trade name, trademark, manufacturer, or otherwise, does not necessarily constitute or imply its endorsement, recommendation, or favoring by the United States Government or any agency thereof or its contractors or subcontractors. The views and opinions of authors expressed herein do not necessarily state or reflect those of the United States Government or any agency thereof. 


\section{Transverse Forces in the First Girder Magnets}

Stephen Brooks

2017-Apr-14

CBETA machine note \#11

\section{Introduction}

These force calculations were done for the Halbach magnets for the CBETA "First Girder" as described in note [CBETA001] and purchased for the assembly of that girder.

\section{QF Magnet}

The picture below shows the transverse forces on each block as vectors (dark red), when the QF magnet is assembled. The light blue arrows are the magnetisation direction.

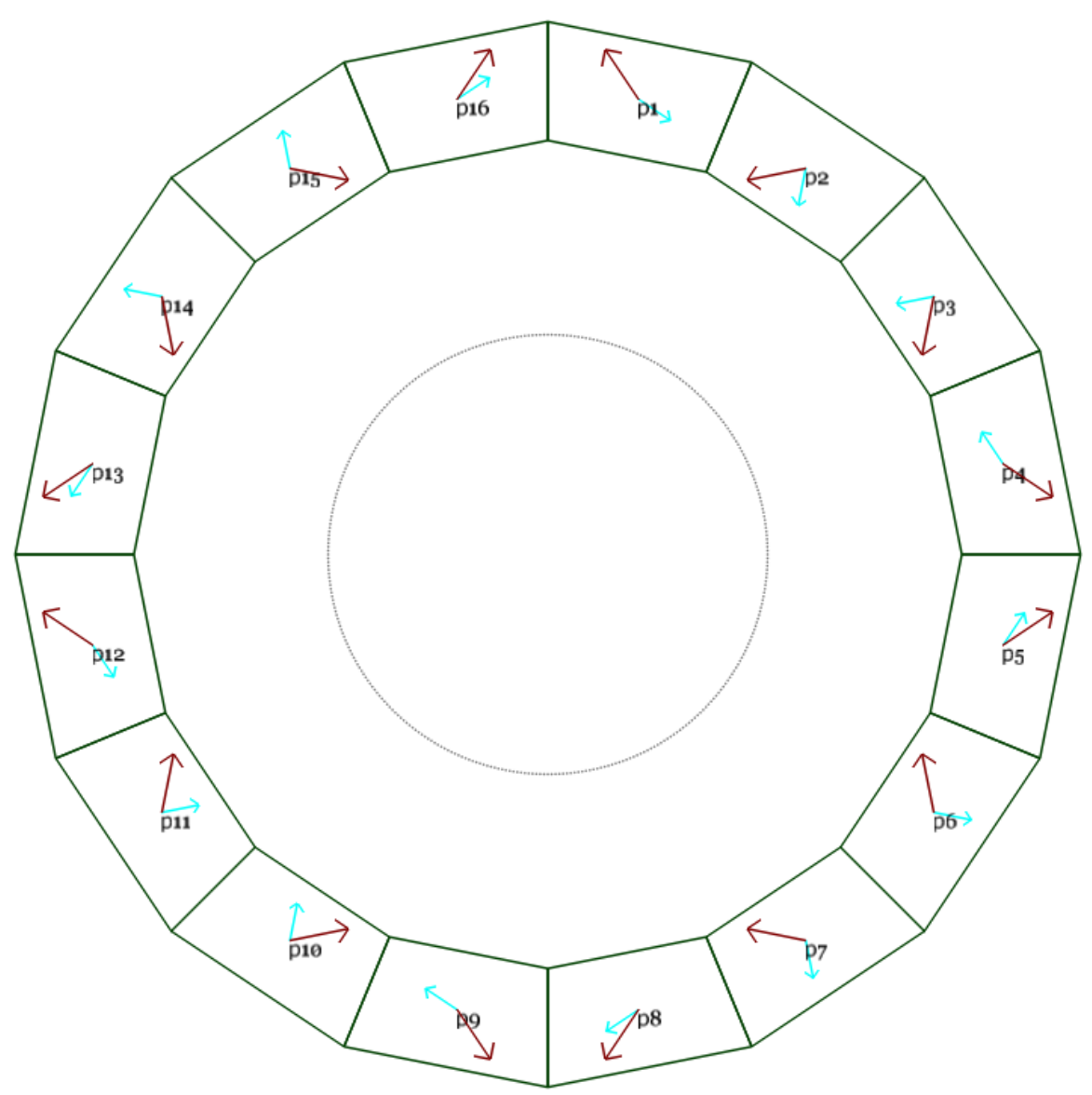

The pieces are labelled arbitrarily p1-p16 so that the corresponding forces can be written in the table below. For actual block type names from the factory, consult the assembly instructions. 
The force can be stated as a force per unit length (Newtons/metre), or a force per block, or for the two blocks constituting the total length of the magnet. The latter gives the force on the outer aluminium shell. ( $F x, F y)$ force vectors are given in each cell.

\begin{tabular}{|l|l|l|l|l|l|}
\hline $\begin{array}{l}\text { Block label in } \\
\text { diagram }\end{array}$ & $\begin{array}{l}\text { Force per unit } \\
\text { length }(\mathrm{N} / \mathrm{m})\end{array}$ & $\begin{array}{l}\text { Force per } \\
\text { block (N) }\end{array}$ & $\begin{array}{l}\text { Force per } \\
\text { block (lbf) }\end{array}$ & $\begin{array}{l}\text { Force full } \\
\text { length (N) }\end{array}$ & $\begin{array}{l}\text { Force full } \\
\text { length (lbf) }\end{array}$ \\
\hline p1 & $(-3470,5177)$ & $(-231,345)$ & $(-52,77.6)$ & $(-463,690)$ & $(-104,155.2)$ \\
\hline p2 & $(-6129,-1228)$ & $(-408,-82)$ & $(-91.8,-18.4)$ & $(-817,-164)$ & $(-183.7,-36.8)$ \\
\hline p3 & $(-1228,-6129)$ & $(-82,-408)$ & $(-18.4,-91.8)$ & $(-164,-817)$ & $(-36.8,-183.7)$ \\
\hline p4 & $(5177,-3470)$ & $(345,-231)$ & $(77.6,-52)$ & $(690,-463)$ & $(155.2,-104)$ \\
\hline p5 & $(5177,3470)$ & $(345,231)$ & $(77.6,52)$ & $(690,463)$ & $(155.2,104)$ \\
\hline p6 & $(-1228,6129)$ & $(-82,408)$ & $(-18.4,91.8)$ & $(-164,817)$ & $(-36.8,183.7)$ \\
\hline p8 & $(-6129,1228)$ & $(-408,82)$ & $(-91.8,18.4)$ & $(-817,164)$ & $(-183.7,36.8)$ \\
\hline p9 & $(-3470,-5177)$ & $(-231,-345)$ & $(-52,-77.6)$ & $(-463,-690)$ & $(-104,-155.2)$ \\
\hline p10 & $(3470,-5177)$ & $(231,-345)$ & $(52,-77.6)$ & $(463,-690)$ & $(104,-155.2)$ \\
\hline p11 & $(6129,1228)$ & $(408,82)$ & $(91.8,18.4)$ & $(817,164)$ & $(183.7,36.8)$ \\
\hline p12 & $(1228,6129)$ & $(82,408)$ & $(18.4,91.8)$ & $(164,817)$ & $(36.8,183.7)$ \\
\hline p13 & $(-5177,3470)$ & $(-345,231)$ & $(-77.6,52)$ & $(-690,463)$ & $(-155.2,104)$ \\
\hline p14 & $(-5177,-3470)$ & $(-345,-231)$ & $(-77.6,-52)$ & $(-690,-463)$ & $(-155.2,-104)$ \\
\hline p15 & $(1228,-6129)$ & $(82,-408)$ & $(18.4,-91.8)$ & $(164,-817)$ & $(36.8,-183.7)$ \\
\hline p16 & $(6129,-1228)$ & $(408,-82)$ & $(91.8,-18.4)$ & $(817,-164)$ & $(183.7,-36.8)$ \\
\hline
\end{tabular}

\section{BD Magnet}

The picture below shows the transvers forces on each block as vectors (dark red), when the BD magnet is assembled. The light blue arrows are the magnetisation direction. 


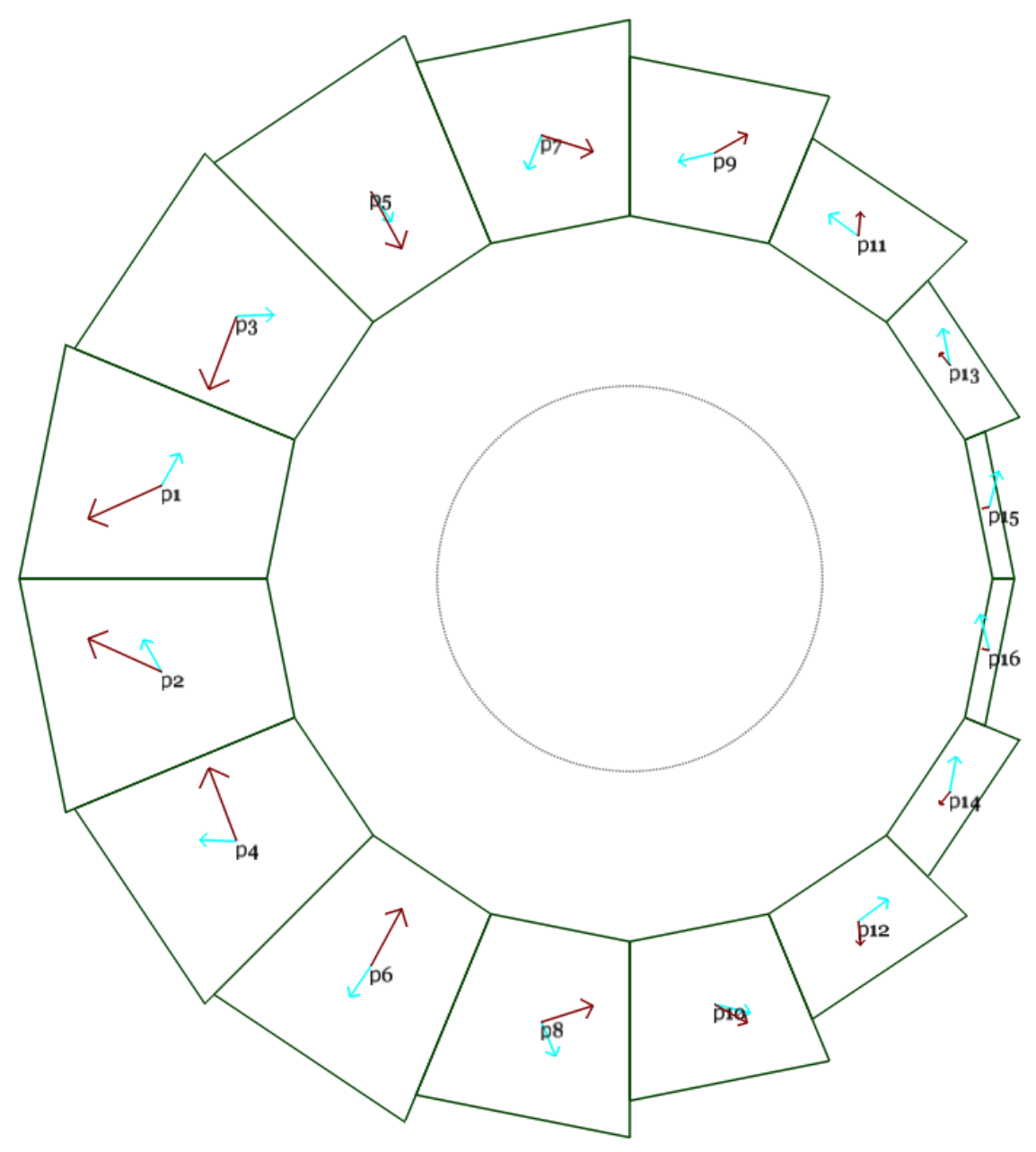

The force vectors on each piece of the BD magnet are written in the table below.

\begin{tabular}{|l|l|l|l|l|l|}
\hline $\begin{array}{l}\text { Block label in } \\
\text { diagram }\end{array}$ & $\begin{array}{l}\text { Force per unit } \\
\text { length }(\mathrm{N} / \mathrm{m})\end{array}$ & $\begin{array}{l}\text { Force per } \\
\text { block (N) }\end{array}$ & $\begin{array}{l}\text { Force per } \\
\text { block (lbf) }\end{array}$ & $\begin{array}{l}\text { Force full } \\
\text { length (N) }\end{array}$ & $\begin{array}{l}\text { Force full } \\
\text { length (lbf) }\end{array}$ \\
\hline p1 & $(-8764,-3967)$ & $(-533,-241)$ & $(-119.9,-54.3)$ & $(-1067,-483)$ & $(-239.8,-108.5)$ \\
\hline p2 & $(-8764,3967)$ & $(-533,241)$ & $(-119.9,54.3)$ & $(-1067,483)$ & $(-239.8,108.5)$ \\
\hline p3 & $(-3330,-8775)$ & $(-203,-534)$ & $(-45.6,-120)$ & $(-405,-1068)$ & $(-91.1,-240.1)$ \\
\hline p4 & $(-3330,8775)$ & $(-203,534)$ & $(-45.6,120)$ & $(-405,1068)$ & $(-91.1,240.1)$ \\
\hline p5 & $(3695,-6845)$ & $(225,-417)$ & $(50.6,-93.6)$ & $(450,-833)$ & $(101.1,-187.3)$ \\
\hline p6 & $(3695,6845)$ & $(225,417)$ & $(50.6,93.6)$ & $(450,833)$ & $(101.1,187.3)$ \\
\hline p7 & $(6137,-1937)$ & $(373,-118)$ & $(83.9,-26.5)$ & $(747,-236)$ & $(167.9,-53)$ \\
\hline p8 & $(6137,1937)$ & $(373,118)$ & $(83.9,26.5)$ & $(747,236)$ & $(167.9,53)$ \\
\hline p10 & $(3909,2199)$ & $(238,134)$ & $(53.5,30.1)$ & $(476,268)$ & $(107,60.2)$ \\
\hline p11 & $(3909,-2199)$ & $(238,-134)$ & $(53.5,-30.1)$ & $(476,-268)$ & $(107,-60.2)$ \\
\hline p12 & $(241,2846)$ & $(15,173)$ & $(3.3,38.9)$ & $(29,346)$ & $(6.6,77.9)$ \\
\hline p13 & $(241,-2846)$ & $(15,-173)$ & $(3.3,-38.9)$ & $(29,-346)$ & $(6.6,-77.9)$ \\
\hline p14 & $(-1170,1438)$ & $(-71,88)$ & $(-16,19.7)$ & $(-142,175)$ & $(-32,39.3)$ \\
\hline p15 & $(-1170,-1438)$ & $(-71,-88)$ & $(-16,-19.7)$ & $(-142,-175)$ & $(-32,-39.3)$ \\
\hline p16 & $(-806,-139)$ & $(-49,-8)$ & $(-11,-1.9)$ & $(-98,-17)$ & $(-22.1,-3.8)$ \\
\hline
\end{tabular}


